\title{
Mortality of pollen grains may result from errors of meiosis: study of pollen tetrads in Typha latifolia L.
}

\author{
VA Berdnikov, OE Kosterin and VS Bogdanova \\ Institute of Cytology and Genetics, Siberian Department of Russian Academy of Sciences, Acad. Lavrentiev ave., 10, Novosibirsk, \\ 630090 Russia
}

In the cattail Typha latifolia the four haploid products of meiosis remain attached and form the flat tetrad of pollen grains. Gametophytic lethals arisen de novo in diploid cells of sporophyte must manifest themselves as pollen tetrads with two dead grains. This could allow to estimate the rate of recessive lethals arresting pollen grain development. We studied pollen samples collected from 44 sprouts in two populations in the vicinity of Novosibirsk. The anomalous tetrads T1, T2, T3, and T4 carrying one, two, three, and four dead grains, respectively, were detected in each sampled individual. The mean frequency of all anomalous tetrads in the two populations was $3.4 \%$ and $8.7 \%$. The frequencies of tetrad classes varied widely among the individuals with correlation coefficient up to 0.94 , but their ratios remained nearly constant. The majority of anomalous tetrads were presented by T1 and T2 classes (their sum comprising 72.7 and $74.0 \%$ in two populations), T1 being a little more abundant. The observed pattern of frequencies of tetrads with dead grains can be explained by errors of male meiosis such as chromosome non-disjunction in both meiotic divisions. The tetrads with two dead pollen grains may result mostly from nondisjunction in anaphase I, and those with one pollen grain from non-disjunction in anaphase II, thus making tetrad analysis ineffective for estimating the rate of gametophytic lethals.

Heredity (2002) 89, 358-362. doi:10.1038/sj.hdy.6800137

Keywords: Typha latifolia L.; tetrad analysis; male gametophyte; mutation rate; chromosome non-disjunction

\section{Introduction}

It is well known that every sample of angiosperm pollen may contain several percent of dead grains, although the reasons of this pollen mortality are insufficiently studied (Drake et al, 1998). After two meiotic divisions a diploid pollen mother cell gives rise to a quartet of haploid microspores, each of which transforms into a haploid pollen grain after a period of growth and two mitotic divisions.

Tetrad formation allows the identification of the reasons for pollen mortality. A microsporocyte heterozygous for a recessive gametophytic lethal should result in a tetrad with two dead grains (Copenhaver et al, 2000), whilst errors of meiosis are also a possible cause for the appearance of dead pollen grains. The first meiotic division separates homologous chromosomes and generates a pair of cells (dyad), therefore, chromosome nondisjunction in meiosis I will result in tetrads with two dead grains. The second meiotic division separates sister chromatids and gives rise to a tetrad of microspores, so that non-disjunction in meiosis II will result in tetrads with one dead grain. The arrest of pollen grain growth for external reasons may account for tetrads with four dead grains. However, as a rule, microspores produced by a microsporocyte separate from each other shortly

Correspondence: OE Kosterin, Institute of Cytology and Genetics, Siberian Department of Russian Academy of Sciences, Acad. Lavrentiev ave., 10, Novosibirsk, 630090 Russia. E-mail: kosterin@bionet.nsc.ru Received 2 July 2001; accepted 8 April 2002 after meiosis and cannot be identified in the pool of mature pollen. Fortunately, in some angiosperms, including Typha latifolia L. (Typhaceae), the members of a tetrad remain attached to each other. As in many monocots, the first meiotic division in T. latifolia is accompanied by wall formation, fixing the position of the cells of a dyad. As a result, all members of the microspore tetrad and, respectively, all grains of the pollen tetrad are arranged in a single plane (Ottaviano and Mulcahy, 1989). This makes it possible not only to estimate the number of dead grains in a tetrad, but also to distinguish adjacent and diagonal arrangements of two abortive pollen grains. In the present study we attempted to estimate the rate and nature of male gametophyte mortality by analyzing the distribution of dead pollen grains in tetrads of $T$. latifolia.

\section{Materials and methods}

Pollen of T. latifolia was collected in two areas $50 \mathrm{~km}$ apart in the vicinity of Novosibirsk (populations $\mathrm{A}$ and B) which are separated by the $\mathrm{Ob}^{\prime}$ River. Population $\mathrm{A}$ (29 samples) consists of five localities: a pond on the Zyryanka brook and four stagnant and semicurrent pools at old oxbows of the right bank floodland of the $\mathrm{Ob}^{\prime}$ River

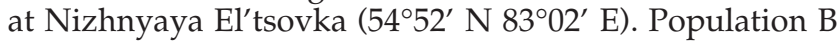
(15 samples) was represented by a small swamp beside a highway crossing a large felled area in a pine forest near Kolyvan' $\left(55^{\circ} 15^{\prime} \mathrm{N} 82^{\circ} 43^{\prime} \mathrm{E}\right)$.

Each sample consisted of pollen taken along the entire length of a single male inflorescence. Pollen samples were placed into hermetically tapped plastic tubes and main- 
tained at $+4^{\circ} \mathrm{C}$. Pollen was stained with acetocarmine (McClintock, 1929), and counts of pollen tetrads were carried out using a $\times 600$ microscope. In total, 210109 tetrads were analyzed (average 4775 per sample).

\section{Results}

Normal pollen tetrads in T. latifolia are flat radially symmetrical isobilateral structures (Figure 1a). Aberrant Tlike or linear tetrads occur rarely, although, a small number of tripartite $(3 p)$ or bipartite $(2 p)$ structures are always present. Pollen grains forming such structures are usually noticeably larger than normal. Large unipartite (1p) spheric structures corresponding in volume to approximately four pollen grains occur extremely rarely. These structures can be interpreted as missing meiotic divisions II ( $3 p$ and $2 p$ ) or I (1p). Estimates of the frequencies of such anomalies in seven plants from population $\mathrm{A}$ showed that $3 \mathrm{p}$ occurred at a frequency of $1.92 \pm 0.29 \%$ of all pollen entities, $2 \mathrm{p}$ at a frequency of $2.58 \pm 0.50 \%$ and $1 p$ at a frequency of $0.04 \pm 0.01 \%$; these structures were excluded from further analysis.

Attention was focused on tetrads containing dead grains which appeared as empty cell walls, and, as a rule,

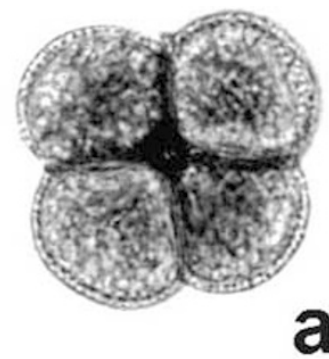

a

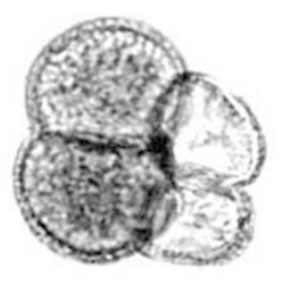

C
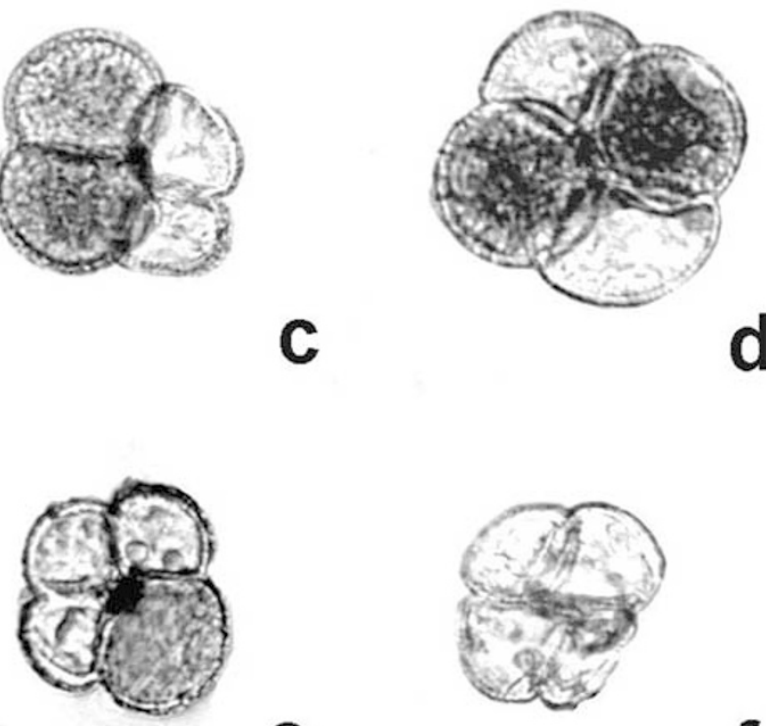

e

\section{$0.05 \mathrm{~mm}$}

Figure 1 The types of pollen tetrads analyzed in Typha latifolia. (a) normal; (b), T1 (one empty grain); (c) T2a (two adjacent empty grains); (d) T2d (two diagonal empty grains); (e) T3 (three empty grains); (f) T4 (four empty grains). empty pollen grains were smaller than viable ones (tetrads containing dead grains are termed 'anomalous'). Mechanically damaged grains were rare and were easily distinguished by their normal size and traces of cytoplasm inside and outside the pollen wall. The frequencies of five classes of anomalous tetrads were estimated: T1 with one empty grain (Figure 1b); T2a - with two adjacent empty grains (Figure 1c); T2d - with two diagonal empty grains (Figure 1d) (the combined class T2a+T2d is labelled T2); T3 - with three empty grains (Figure 1e); and T4 - with four empty grains (Figure 1f).

In some samples there were pollen clumps consisting entirely of empty tetrads. Probably, in these cases all the anther contents perished for some reason. Such clumps were excluded from the analysis. Some T4 tetrads may have originated from dead anthers, as implied by somewhat higher standard deviation of the $\mathrm{T} 4$ frequencies in comparison with other classes (Table 1).

For seven plants from population A we compared pollen samples taken from base to top along a male inflorescence and found no trend in the frequencies of all tetrad classes (not presented).

The mean frequency of all anomalous tetrads in the two populations is $3.4 \%$ and $8.7 \%$ (Table 1 ). The majority of anomalous tetrads are represented by two classes, T1 and $\mathrm{T} 2$, comprising in sum $72.7 \%$ and $74.0 \%$ in populations A and B, respectively, T2 being a little less abundant. The distributions of frequencies of all the types of anomalous tetrads in population B are substantially shifted to larger values as compared to those of population A (Figure 2). The mean frequency of empty pollen grains among all types of tetrads is $1.79 \pm 0.16 \%$ in population $\mathrm{A}$ and $4.32 \pm 0.64 \%$ in population B (Table 1).

The frequencies of all anomalous tetrad classes varied concertedly over the samples studied. Table 2 gives correlation coefficients of frequencies of the tetrad classes for all samples of the two populations. All correlations are highly significant, the frequency of the T4 class displays the lowest correlation with the others. The graph of Figure 3 illustrates a strong correlation between the frequencies of the tetrad classes T1 and T2.

Table 1 Mean frequencies and standard deviations of tetrad classes T1, T2, T3, T4, all anomalous tetrads (TT) and the frequencies of dead pollen grains among pollen tetrads ( $\mathrm{pl}$ ) for populations $\mathrm{A}$ and B. $n=$ number of plants studied

\begin{tabular}{lccccc}
\hline \multirow{2}{*}{$\begin{array}{c}\text { Tetrad } \\
\text { classes }\end{array}$} & \multicolumn{2}{c}{$\begin{array}{c}\text { Population } \\
n=29\end{array}$} & & \multicolumn{2}{c}{$\begin{array}{c}\text { Population } B \\
n=15\end{array}$} \\
\cline { 2 - 3 } \cline { 6 - 6 } & mean \pm st.error & std.dev & & mean \pm st.error & std.dev \\
\hline T1 & $0.0131 \pm 0.0016$ & 0.0236 & & $0.0368 \pm 0.0061$ & 0.0088 \\
T2a & $0.0097 \pm 0.0010$ & 0.0094 & & $0.0221 \pm 0.0024$ & 0.0054 \\
T2d & $0.0020 \pm 0.0003$ & 0.0055 & & $0.0053 \pm 0.0014$ & 0.0015 \\
T2 & $0.0116 \pm 0.0012$ & 0.0142 & & $0.0274 \pm 0.0037$ & 0.0067 \\
T3 & $0.0021 \pm 0.0004$ & 0.0066 & & $0.0093 \pm 0.0017$ & 0.0020 \\
T4 & $0.0072 \pm 0.0009$ & 0.0089 & & $0.0133 \pm 0.0023$ & 0.0046 \\
TT & $0.0341 \pm 0.0033$ & 0.0177 & & $0.0868 \pm 0.0130$ & 0.0503 \\
pl & $0.0179 \pm 0.0016$ & 0.0250 & & $0.0432 \pm 0.0064$ & 0.0085 \\
\hline
\end{tabular}

Total number of tetrads counted in populations A and B were 154,702 and 55,407, respectively. 


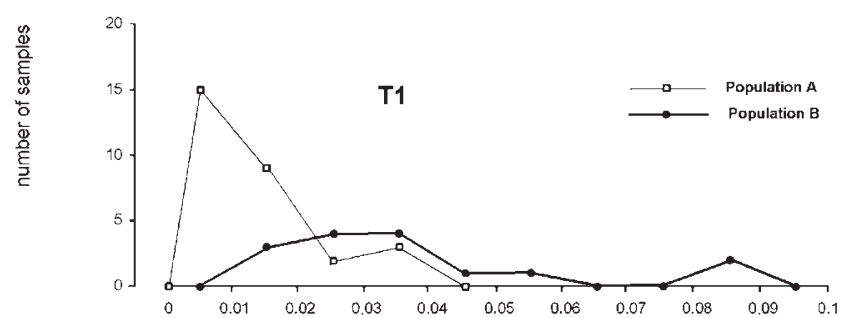

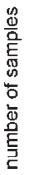
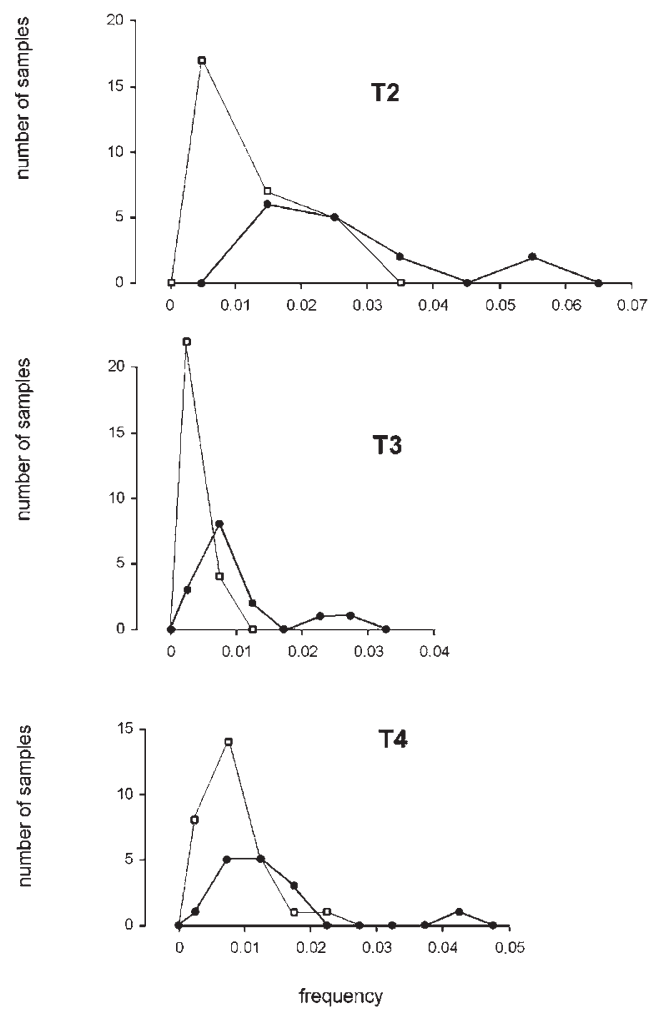

Figure 2 Distributions of frequencies of anomalous tetrads of the $\mathrm{T} 1, \mathrm{~T} 2, \mathrm{~T} 3$ and T4 classes in pollen samples of populations A and B of Typha latifolia, with 29 and 15 plants analyzed, respectively.

Table 2 Correlation coefficients among frequencies of the pollen tetrad classes T1, T2, T3, and T4 over all plants studied

\begin{tabular}{lcccc}
\hline & T2a & T2d & T3 & T4 \\
\hline T1 & 0,886 & 0,901 & 0,951 & 0,635 \\
T2a & & 0,810 & 0,884 & 0,590 \\
T2d & & & 0,867 & 0,572 \\
T3 & & & & 0,737
\end{tabular}

For all coefficients, probability of correlation absence $P<0.5 \%$.

\section{Discussion}

Our results can be summarized as follows: (i) T2 class that could be explained by an action of gametophytic lethals comprises only about $30 \%$ of anomalous tetrads being less abundant than the T1 class; (ii) the relative proportions of classes $\mathrm{T} 1, \mathrm{~T} 2 \mathrm{a}, \mathrm{T} 2 \mathrm{~d}$ and $\mathrm{T} 3$ remain very close in the two populations studied in spite of large variation of the total ratio of anomalous tetrads in pollen samples. A common mechanism underlying the characteristic distribution of frequencies of anomalous tetrads must exist.

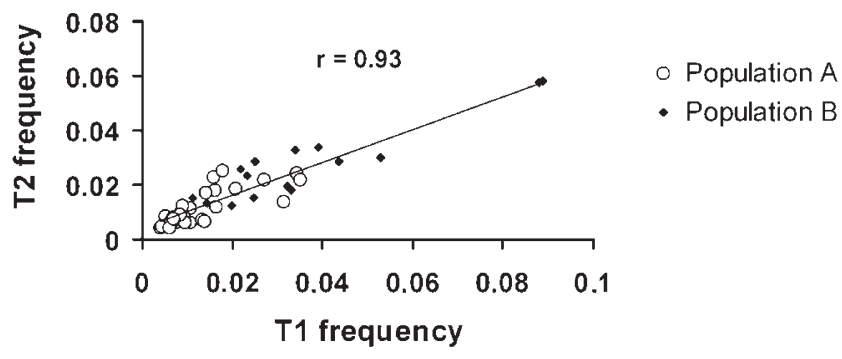

Figure 3 Frequencies of T2 tetrads of Typha latifolia plotted against frquencies of T1 tetrads in the samples of populations A (empty circles) and B (filled diamonds).

In the present work pollen was analyzed from 44 plants of two populations separated by a distance of about $50 \mathrm{~km}$ and the $\mathrm{Ob}^{\prime}$ River. The mean proportion of empty pollen grains among all the grains counted was $1.8 \%$ in population A and $4.3 \%$ in population B. Typha latifolia is a perennial plant that is propagated primarily by rhizomes. The observed difference in the mean proportion of empty pollen grains could be attributed to differences in mean rhizome age so that an older population could have accumulated more gametophytic lethals. However, population B seems to be more recent since it appeared in a small pool near a highway constructed not earlier than 50 years ago, whilst population A exists in old floodland swamps.

Dozens of years and thousands of cell generations pass from seed germination to meiosis in an inflorescence. Thus, it is natural to expect sporophytic cells of rhizomes to accumulate and maintain in heterozygous state the gametophytic lethals which should then be revealed as pollen tetrads with two empty pollen grains. Large clones of diploid cells carrying mutations could have included an entire inflorescence or its large part. However, none of the 44 samples studied had a frequency of T2 tetrads that exceeded $6 \%$, indicating the small size of cell clones. This unexpectedly low rate of gametophytic mutations killing pollen grains may be explained by some dominance of these mutations, so that cell clones heterozygous for null alleles are substituted by clones of normal sporophytic cells.

Strong correlation among frequencies of the classes of anomalous pollen tetrads indicates that their origins are based on a common mechanism which acts during meiosis. Note that in the mouse about a half of spontaneous mutations takes place during 'perigametic' interval from the premeiotic DNA replication to the first division of the zygote (Russel, 1999). In addition, it is known that meiotic crossing-over between non-allelic repeated sequences of homologous or sister chromatids can produce deletions (Shaffer and Lupsky, 2000), that might kill one pollen grain of a tetrad. However, the rate of spontaneous deletions in maize (Brock and Pryor, 1996) and man (Shaffer and Lupsky, 2000) does not exceed $10^{-3}$ per genome per generation and cannot account for the observed high frequency of dead pollen grains and the similarity of $\mathrm{T} 1$ and $\mathrm{T} 2$ frequencies.

At the same time, in heterozygotes for translocation, about $50 \%$ of pollen grains perish because of loss of a considerable portion of genome in a half of meiocytes due to imbalanced disjunction of chromosomes in anaphase I. The high frequencies of dead pollen grains 
observed in T. latifolia may be caused by some errors of meiosis resulting in chromosome mis-segregation.

Transformation of a pollen mother cell into four microspores involves three anaphase events, one in meiosis I and two in meiosis II. If it is assumed that nullisomy for a chromosome is lethal to a developing gametophyte while disomy is tolerable, non-disjunction in the first division will produce $\mathrm{T} 2 \mathrm{a}$ tetrads, whereas non-disjunction in the second division will result in T1 tetrad (Figure 4). T2d tetrads should result from two non-disjunction events that occurred in both dyad cells. T3 tetrads can be caused by two successive non-disjunction events; the first at anaphase I and the second at anaphase II of a dyad cell which received a nonseparated bivalent. As a result, three microspores of the tetrad lacking a portion of the genome will die. T4 tetrads can appear due to the loss of a bivalent lagged during non-disjunctional anaphase I. This mechanism implies correlation of the frequency of T4 tetrads with those of other anomalous tetrads. At the same time, clumps of dead tetrads suggest that some factors occasionally cause local pollen death, which could explain the relatively weak correlation of $\mathrm{T} 4$ frequency with those of T1, T2a and T2d.

If the probability of non-disjunction in a meiotic division is small (less than 0.05), the frequency of T2a tetrads should be close to the probability of non-disjunction in anaphase I $\left(\mathrm{p}_{1}\right)$ whilst the frequency of T1 tetrads should be twice as high as the probability of non-disjunction in anaphase II of each dyad cell $\left(\mathrm{p}_{2}\right)$. From Table 1 one can infer that $p_{1}=0.010$ and $p_{2}=0.007$ for population $\mathrm{A}$ and $\mathrm{p}_{1}=0.022$ and $\mathrm{p}_{2}=0.018$ for population B. However, the frequencies of $\mathrm{T} 2 \mathrm{~d}$ and $\mathrm{T} 3$ tetrads resulting from two independent non-disjunction events will be at least an order of magnitude lower then the observed ones (Figure 4). This discrepancy can be explained if we suppose that in some male meiocytes the control over fidelity of chromosome segregation in both meiotic divisions is weakened. If we assume that anomalous tetrad classes $\mathrm{T} 1, \mathrm{~T} 2 \mathrm{a}, \mathrm{T} 2 \mathrm{~d}$, and $\mathrm{T} 3$ result from such anomalous meiocytes and accept, for simplicity, that $\mathrm{p}_{1}=\mathrm{p}_{2}=1 / 3$, pro-

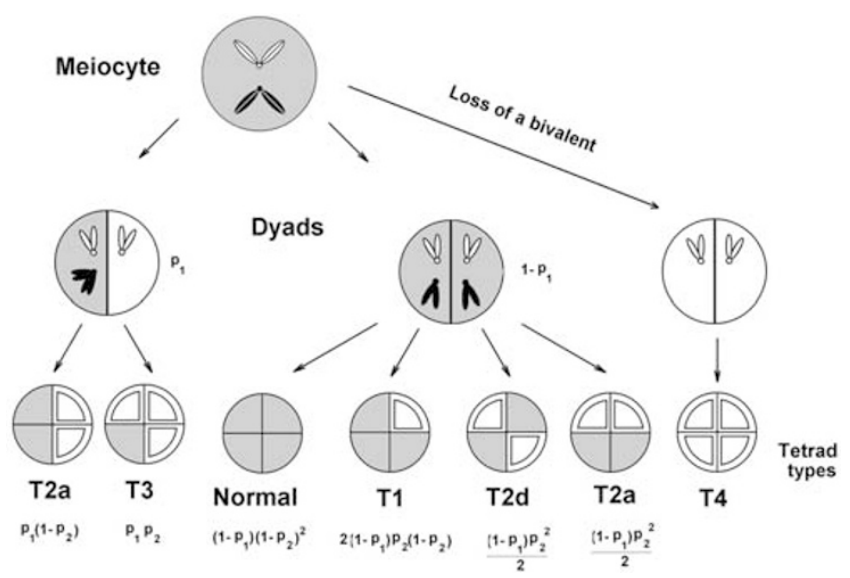

Figure 4 A scheme of origin of different classes of pollen tetrads with dead grains by chromosome non-disjunction in meiosis I and II. Probabilities of tetrad types are given under the relevant diagrams assuming that no bivalent loss takes place and no more than one non-disjunction event occurs in any anaphase. $\mathrm{p}_{1}$ and $\mathrm{p}_{2}$ are probabilities of non-disjunction in anaphase I and II, respectively. portions of these classes (Figure 5c) are obtained that are not far from the observed ones (Figure $5 \mathrm{a}, \mathrm{b}$ ); the probability of a bivalent loss, leading to appearance of $\mathrm{T} 4$ tetrads not considered. The observed frequencies can be obtained if we assume the mean share of anomalous meiocytes with weakened control of chromosome segregation as $\sim 4 \%$ for population $\mathrm{A}$ and $\sim 10 \%$ for population $\mathrm{B}$, that is not far from the observed proportion of anomalous tetrads in these populations (Table 1).

A simple Monte-Carlo computer model has been investigated in which non-disjunction events involving each segregating unit (pair of homologs in division I or pair of chromatids in division II) occur with equal probability. 50,000 trials with a probability of 0.02 for each of 15 bivalents (in T. latifolia, $2 n=30$, Darlington and Wylie, 1955) lead to proportions of classes T1, T2a, T2d and T3 fairly close to observed ones (Figure $5 \mathrm{~d}$ ). In this model T4 tetrads can arise from non-disjunction of two bivalents in anaphase I of the same meiocyte resulting in nullisomy of both daughter cells of the dyad. With the above parameters, the proportion of T4 slightly exceeds that of T2d.

Tri-, bi- and monopartite structures present among tetrads most probably result from a failure of some meiotic divisions and so provide additional evidence that anomalies of male meiosis are common in the cattail. Meiosis is a very complicated process the proper course of which is governed by many genes with finely tuned activities (eg, Golubovskaya, 1989). It is also known that the frequency of non-disjunction is influenced by external factors such as temperature in Drosophila (Ashburner, 1989) or mother age in Drosophila (Ashburner, 1989) and man (Vogel and Motulsky, 1986). The appearance of meiocytes with weakened control over chromosome segregation in both meiotic divisions may result from many causes, for example, loosening cohesion of sister chromatids holding together homologs at metaphase I and sister chromatids at metaphase II (Roeder, 1997; Dawe, 1998) or defects of the spindle apparatus. For this reason, the difference in the frequency of anomalous tetrads between two T. latifolia populations can be accounted for by some environmental factors or by difference in genetic background.

a
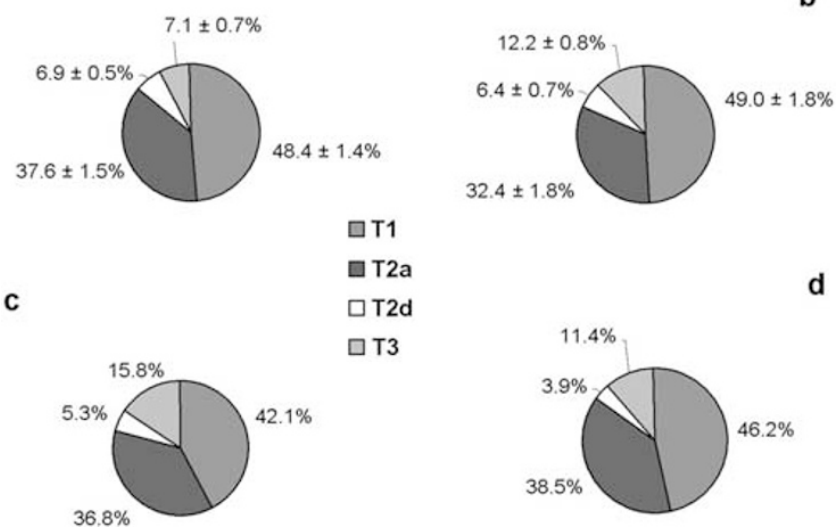

Figure 5 Mean proportions of T1, T2a, T2d, T3 tetrads in the sum of these classes in populations A (a) and B (b) of Typha latifolia and the theoretically expected ones: (c) calculated from probabilites of Figure 4 assuming no more than one non-disjunction event per anaphase cell and $p_{1}=p_{2}=1 / 3,(d)$ obtained by a Monte-Carlo model with no limitation on the number of non-disjunctions per anaphase cell and probability $\mathrm{p}_{1}=\mathrm{p}_{2}=0.02$ per bivalent in anaphases I and II. 


\section{Acknowledgement}

We express our sincere gratitude to Dr Alexey Kondrashov for encouraging us in this study, fruitful discussion and critically reading the manuscript.

\section{References}

Ashburner M (1989). Drosophila. A. Laboratory Handbook. Cold Spring Harbor Laboratory Press: Cold Spring Harbor.

Brock RD, Pryor AJ (1996). An unstable minichromosome generates variegated oil yellow maize seedlings. Chromosoma 104: 575-584.

Copenhaver GP, Keith KC, Preuss D (2000). Tetrad analysis in higher plants. A budding technology. Plant Physiol 124: 7-16.

Darlington CP, Wylie AP (1955). Chromosome Atlas of Flowering Plants. George Allen and Unwin Ltd: London.

Dawe RK (1998). Meiotic chromosome organization and segregation in plants. Annu Rev Plant Physiol Plant Mol Biol 49: 371-395.
Drake JW, Charlesworth B, Charlesworth D, Crow JF (1998). Rates of spontaneous mutation. Genetics, 148: 1667-1686.

Golubovskaya IN (1989). Meiosis in maize: mei genes and conception of genetic control of meiosis. Adv Genet 26: 149-192.

McClintock B (1929). A method for making aceto-carmine smears permanent. Stain Technol 4: 53-56.

Ottoviano E, Mulcahy DL (1989). Genetics of angiosperm pollen. Adv. Genet. 26: 1-64.

Roeder GS (1997). Meiotic chromosomes: it takes two to tango. Genes Develop 11: 2600-2621.

Russel LB (1999). Significance of the perigametic interval as a major source of spontaneous mutations that result in mosaics. Environ Mol Mutagen 34: 16-23.

Shaffer LG, Lupsky JR (2000). Molecular mechanisms for constitutional chromosomal rearrangements in humans. Annu Rev Genet 34: 297-329.

Vogel F, Motulsky AG (1986). Human Genetics. Problems and Approaches. Springer-Verlag: Berlin, Heidelberg, New York, Tokyo. 